\title{
Chaotic motion of relativistic electrons driven by whistler waves
}

\author{
G V Khazanov', A A Tel'nikhin', and Tatiana K. Kronberg ${ }^{2}$ \\ ${ }^{1}$ NASA Marshall Space Flight Center, National Space Science \& Technology Center, \\ 320 Sparkman Drive, Huntsville, Alabama 35805, USA \\ 2 Altai State University, Dimitrov, 66, Barnaul 656049, Russia \\ 2 Altai State University, Dimitrov, 66, Barnaul 656049, Russia \\ E-mail: tatiana.kronberg@list.ru
}

PACS numbers: $52.65, \mathrm{Cc} 52.20, \mathrm{Dq} 05.45, \mathrm{Pq}, 94.20, \mathrm{Rr}$ 


\begin{abstract}
Canonical equations governing an electron motion in electromagnetic field of the whistler mode waves propagating along the direction of an ambient magnetic field are derived. The physical processes on which the equations of motion are based are identified. It is shown that relativistic electrons interacting with these fields demonstrate chaotic motion, which is accompanied by the particle stochastic heating and significant pitch angle diffusion. Evolution of distribution functions is described by the Fokker-Planck-Kolmogorov equations. It is shown that the whistler mode waves could provide a viable mechanism for stochastic energization of electrons with energies up to $50 \mathrm{MeV}$ in the Jovian magnetosphere.
\end{abstract}

\title{
1. Introduction
}

A description of the stochastic dynamics of charged particle in the field of wave packet is one of the fundamental problem in the theory of plasma physics, that has attracted current interest for many years [1]. Thus the stochastic dynamics of relativistic electrons in the time-like wave packet has been discussed by Chernikov et al [2]. A theory of dynamic chaos has also been employed to deal with high-frequency heating of electrons in the Langmuir wave packet [3]. Possible astrophysical applications of this results were discussed in [4]. Nagornykh et al have been announced some results of theoretical study concerning stochastic particle motion in the magnetized plasmas [5]. On the other hand, Zaslavsky et al [6] have been reported the results describing electron stochastic motion in the upper-hybrid wave propagating across an ambient magnetic field. Concepts and methods which were developed in these works have impacted our approach to the problem.

The goal of present paper is a systematic study of relativistic particle motion in a whistler wave packet. Earlier the basic concepts of energy diffusion of relativistic electrons resulting from resonant interaction with whistlers in the magnetosphere have been discussed, for example, by Walker [7], and Summers et al [8]. In our work we discuss stochastic motion of high-energy electrons due to a spectrum of whistler mode waves. The results of study of the problem are of interest from the point of view interpreting the experimental results.

The paper is organized as follows. The canonical equation of motion expressed in terms of the action- angle variables are derived in Sec.2. The nonlinear resonance wave-particle interaction is discussed qualitatively in Sec.3. In Sec.4 it is shown that the stochastic motion of relativistic electrons can be described by the closed set of nonlinear difference equations. The solutions of these equations are obtained both analytically and numerically. In Sec.5 it is proved that particle dynamics is realized on stochastic attractor with fractal structure. The Fokker-Planck-Kolmogorov equation is derived in Sec.6, and the effects associated with particle stochastic heating such that the pitch angle scattering and radial drift of plasma particles are studied. Application of our results to high-energy electrons observed in the Jovian election radiation belts is described in Sec.7. In Sec. 8 we give the conclusions of our studies. 


\section{Basic equations}

Let us consider a relativistic particle of charge $|e|$ and mass $m$ in the wave packet of extraordinary electromagnetic waves propagating along an external uniform magnetic field of strength $B$. The Hamiltonian corresponding to the problem is

$$
H(\mathbf{r}, \mathbf{p} ; t)=\sqrt{m^{2}+(\mathbf{p}+\mathbf{A})^{2}}
$$

and the canonical equations of motion are

$$
\dot{\mathbf{p}}=[\mathbf{p}, H], \quad \dot{\mathbf{r}}=[\mathbf{r}, H],
$$

where $\mathbf{p}$ is the canonical momentum, $\mathbf{r}$ is the position vector, $\mathbf{A}$ the vector potential divided into two parts, $\mathbf{A}=\mathbf{A}^{w}+\mathbf{A}^{\text {ext }}$, superscripts $w$ and ext denote both the wave and external fields, and [, ] stand for the Poisson brackets.

We have employed here and throughout this paper, the frame of reference in which the speed of light $c=1$ and charge $|e|=1$.

We denote by $R$ the set of all real numbers. Then $\mathbf{p} \in R^{3}, \mathbf{r} \in R^{3}$, and the smooth manifold $M=R^{6}$ will be a canonical space of this dynamic system, and $R^{6}=R^{3} \times R^{3}$ is a direct product space.

In order to write down equations of the particle motion one must specify a coordinate system. We have chosen a Cartesian spatial coordinates system whose $z$ axis is directed along the external magnetic field, the plane perpendicular to this direction is spanned by the orthogonal coordinates $x$ and $y$.

Making use of the connecting relations,

$$
\mathbf{B}=[\nabla, \mathbf{A}], \quad \mathbf{E}=-\partial \mathbf{A} / \partial t
$$

we have in the coordinate representation

$$
\begin{aligned}
& \mathbf{r}=(x, y, z), \quad \mathbf{B}^{e x t}=(0,0, B), \\
& \mathbf{A}^{w}=\left(\sum_{k} A_{k} \sin \varphi, \sum_{k} A_{k} \cos \varphi, 0\right), \\
& \varphi \quad=z k-t \omega_{k}, \\
& \mathbf{A}^{e x t}=(-B y, B x, 0) / 2 .
\end{aligned}
$$

Here the expression for $\mathbf{A}^{e x t}$ is written in the axial gauge, $A_{k}$ is the amplitude of mode in the wave packet, $k$ is the wave number, and $\omega_{k}$ is the dispersion equation.

The dispersion relation for electron branch of the whistler mode waves in the cold magnetoplasma is written as

$$
k^{2} / \omega^{2}=1+\omega_{p}^{2} /\left[\omega\left(\omega_{B}-\omega\right)\right]
$$

where $\omega_{B}$ and $\omega_{p}$ are the gyrofrequency and electron plasma frequency, respectively. Equation (7) in a weak magnetic field when the condition $\left(\omega_{B} \omega / \omega_{p}^{2}\right) \ll 1$ is valid, reduces to

$$
v_{p h}^{2}=\frac{\omega\left(\omega_{B}-\omega\right)}{\omega_{p}^{2}}, \quad v_{p h}^{2} \ll 1
$$


In a strong magnetic field, provided that $\omega / \omega_{B} \simeq \omega_{p} / \omega_{B}, \omega_{p}^{2} /\left(\omega \omega_{B}\right) \ll 1$, equation (7) reduces to

$$
v_{p h}=1-\frac{\omega_{p}^{2}}{2 \omega \omega_{B}}
$$

We now take into account axial symmetry of the non-perturbative problem, and introduce the new variables, an action $(I)$, and an angle $(\theta)$, by a canonical transformation $\left(x, p_{x} ; y, p_{y}\right) \rightarrow(\theta, I):$

$$
\begin{array}{ll}
x=r \cos \theta, & p_{x}=-\left(m r \omega_{B} / 2\right) \sin \theta, \\
y=r \sin \theta, & p_{y}=\left(m r \omega_{B} / 2\right) \cos \theta ; \\
r=\sqrt{2 m \omega_{B} I} / m \omega_{B}, \omega_{B}=B / m,
\end{array}
$$

where $r$ is the gyroradius.

The Hamiltonian (1) in this representation becomes

$$
\begin{aligned}
H\left(z, p_{z} ; \theta, I ; t\right)= & H_{0}(p, I)+\sqrt{2 m \omega_{B} I} H_{0}^{-1} \\
& \cdot \sum_{k} A_{k} \cos \left(z k+\theta-t \omega_{k}\right), \\
H_{0}(p, I)= & \sqrt{m^{2}+p^{2}+2 m \omega_{B} I}, \quad p_{z} \equiv p .
\end{aligned}
$$

Here we have assumed that the ratio $\mu=A^{w} / m \ll 1$ is the small parameter of the problem, and retain in (12) only the leading terms.

Associated with (12) the equations of motion are

$$
\begin{aligned}
& \dot{p}=[p, H]=\sqrt{2 m \omega_{B} I} H_{0}^{-1} \cdot \sum_{k} k A_{k} \sin \psi, \\
& \dot{I}=[I, H]=\sqrt{2 m \omega_{B} I} H_{0}^{-1} \cdot \sum_{k} A_{k} \sin \psi, \\
& \dot{z}=[z, H]=p H_{0}^{-1}, \quad \dot{\theta}=[\theta, H]=\omega_{B} m H_{0}^{-1} .
\end{aligned}
$$

We omit in (16) the terms of the order of $\mu^{2}$ and introduce the definition for the phase

$$
\psi \stackrel{\text { def }}{=} z k+\theta-\omega_{k} t \text {. }
$$

It is obvious that a Hamiltonian flow $(15,16)$ preserves the measure $d \mu=d p_{z} d I d z d \theta$ on the smooth manifold $M=R^{3} \times S$, where $S$ is the circle and $\theta(\bmod 2 \pi) \in S$. Two pairs of the canonical variables $p, z$ and $I, \theta$ are a symplectic coordinates on $M$ with that algebra:

$$
\begin{aligned}
& {\left[e_{p}, e_{\theta}\right]=\left[e_{p}, e_{I}\right]=\left[e_{I}, e_{z}\right]=\left[e_{z}, e_{\theta}\right]=0,} \\
& {\left[e_{p}, e_{z}\right]=\left[e_{I}, e_{\theta}\right]=1,}
\end{aligned}
$$

where [,] denote a skew-product, the set $\left(e_{j}\right)$ is the coordinate basic, which can be given by a differential operators $\partial / \partial x^{j}$ (Schutz, 1982).

This algebra will be used in the following. 
Now the structure of wave packet, $A^{w}(t, z)=\sum_{k} A_{k} \exp \left[i\left(z k+\theta-t \omega_{k}\right)\right]$, is to be specialized. The most frequently used representations of wave packet are the so-called time- and space-like representations [6], [9]. Such wave packet may be exited in a plasma due to the intrinsic instabilities. In this particular physical situation, we make following simplifying assumptions regarding the structure of the packet,

$$
k=k_{0}+n \Delta k, \quad \omega=\omega_{0}+n \Delta \omega, \quad \Delta k / k_{0} \ll 1, \quad \Delta \omega / \omega_{0} \ll 1, \quad n \in Z,
$$

where $k_{0}, \omega_{0}$ are the characteristic wave number and frequency, $\Delta \omega(\Delta k)$ is the group dispersion in wave spectrum, so that

$$
\Delta \omega=v_{g r} \Delta k, \quad v_{g r}=d \omega / d k,
$$

$v_{g r}$ is the group velocity,

$$
\Delta k=2 \pi / L, \Delta \omega=2 \pi / T
$$

$L, T$ are the length- and the time- scales of the problem, $Z$ denotes the set of all integers. Then we suppose that the characteristic spectral amplitude $A_{0}$ is a slowly varying function on $t$ and $z$ such that

$$
\dot{A}_{0} / \omega A_{0} \simeq 1 / \omega T(\ll 1), \quad \nabla A_{0} / k A_{0} \simeq 1 / k L(\ll 1),
$$

and write down the wave packet in the form

$$
\begin{aligned}
& A^{w}(t, z)=A_{0} \exp \left(i \psi_{0}\right) \sum_{n \in Z} A_{k} \exp [i(n \Delta k z+n \Delta \omega t)], \\
& \psi_{0}=k_{0} z+\left(\omega_{B} m H_{0}^{-1}-\omega_{0}\right) t .
\end{aligned}
$$

Define the parameter, $\eta$ namely, the ratio of the particle velocity along an ambient magnetic field to the group velocity,

$$
\eta=v_{z} / v_{g r}
$$

In the limit, $\Delta k \rightarrow 0, \eta \rightarrow 0$, expression (22) can be transformed into

$$
A^{w}(t, z)=A_{0} \exp \left(i \psi_{0}\right) \sum_{n \in Z} \delta(t / T-n)
$$

There is the time-like representation (TLR) of a wide wave packet. The Poisson sum formula

$$
\sum_{n \in Z} \exp (i n \Delta \omega t)=\sum_{n \in Z} \delta(t / T-n)
$$

has employed in (25), where $\delta(\cdot)$ is the Dirac delta-function.

In another limit $\Delta \omega \rightarrow 0, \eta \rightarrow \infty$, we can easily show that the wave field takes the form of the space-like (SL) wave packet

$$
A^{w}(t, z)=A_{0} \exp \left(i \psi_{0}\right) \sum_{n \in Z} \delta(z / L-n)
$$

This packet represents a periodic sequence of impulses with characteristic spatial period $L=2 \pi / \Delta k$. 
Note that both representation are often used. Thus it is established [6], [9], that the TLR is available for the problem if the condition $\eta^{2} \ll 1$ holds. The TLR of the electric field of the electrostatic waves was used by Chernikov et al [2] to derive the relativistic generalization of the standard map. On the other hand, the SLR has been utilized in [3] to describe the stochastic motion of relativistic particle in the electrostatic field of Langmuir waves, whose group velocity is small as known.

\section{Qualitative analysis of the particle motion}

We now proceed to the physical aspect of the problem. First we consider the dynamics of an autonomous system with the Hamiltonian given by (13). In this commutative integrable situation $(\dot{I}=0, \quad \dot{p}=0)$, the phase curves lie on constant energy surfaces of $H_{0}=E=$ const:

$$
\left\{(z, p ; \theta, I): \sqrt{m^{2}+p^{2}+2 m \omega_{B} I}=E\right\} .
$$

All solutions are regular, since trajectories in phase space cannot intersect and each phase curve correspond in configuration space to particle gyration in an external magnetic field with a frequency $\omega_{B} m H_{0}^{-1}$.

To understand the physical picture of the stochastic motion, let us discuss the simple case of the single-wave Hamiltonian,

$$
H=H_{0}(p, I)+\sqrt{2 m \omega_{B} I} H_{0}^{-1} \cdot A_{k} \cos \left(z k+\theta-\omega_{k} t\right),
$$

where $A_{k}$ is the amplitude of certain mode in the wave packet, $k$ is an arbitrary wavenumber, $\omega_{k}$ is the frequency of this mode.

Thus the associated equations of motion are

$$
\begin{aligned}
& \dot{p}=k \sqrt{2 m \omega_{B} I} H_{0}^{-1} \cdot A_{k} \sin \psi, \\
& \dot{I}=\sqrt{2 m \omega_{B} I} H_{0}^{-1} \cdot A_{k} \sin \psi, \\
& \dot{z}=v_{z}=p H_{0}^{-1}, \quad \dot{\theta}=\omega_{B} m H_{0}^{-1} \\
& \dot{\psi_{k}}=\omega(k, E)=k v_{z}+\omega_{B} m E^{-1}-\omega_{k},
\end{aligned}
$$

$\omega(k, E)$ is the nonlinear phase frequency.

It is easy to see that the system possesses the first integral, $i \dot{n} v .=(\dot{p}-k \dot{I})=0$. We put const. $=0$ below and represent the invariant of motion as

$$
\alpha p-2 \omega_{B} I=0, \quad \alpha=\frac{2 \omega_{B}}{\omega} v_{p h} .
$$

It is well-known [1] that the stochastic motion will be mainly determined by the nonlinear resonance wave-particle interaction,

$$
\omega\left(k, E_{r}\right)=\left(k v_{z}+\omega_{B} m E^{-1}-\omega_{k}\right)_{r}=0,
$$

as well as the group velocity dispersion.

In the wave spectrum case the equation given above determines the family of resonance states in energy space. The phase space of resonance states has structure of the product 
bundle, $B \times F$, where $B$ is the base, i.e. the open subset $U \subset R^{2}, I_{r}, p_{r} \in U$, and $F$ is the typical fibre, $F \cong T^{2}$, with the coordinates $\left(\left(\omega-k v_{z}\right) t, \theta(\operatorname{modd} 2 \pi)\right) \in T^{2}$.

Now varying of (34) with respect to $\omega, k, E_{\tau}$ we obtain the interval between an adjacent fibres,

$$
\Delta E=\left(v_{z} \Delta k-\Delta \omega\right) /\left(k\left(\partial v_{z} / \partial E\right)_{r}-\omega_{B} m E^{-2}\right)
$$

Here $\Delta k$ and $\Delta \omega$ are the intervals between an adjacent modes in the wave spectrum, and $\Delta E$ is the interval between the two adjacent resonance states in the particle energy space.

We specify the problem. First we introduce the following notation

$$
\varepsilon \equiv E / m, \quad \varepsilon_{z} \equiv p / m, \quad \varepsilon_{t} \equiv \sqrt{2 m \omega_{B} I} / m
$$

and denote by $\sup \{\varepsilon\}=\varepsilon_{b}$ the upper bound of the set $\{\varepsilon\}$.

On condition that (8) is valid, and the parameter $\alpha$ is small, we stipulate

$$
\varepsilon_{b}^{2} \gg \max \left\{\alpha^{2}, 1\right\}
$$

unlike this case, we assume that the condition

$$
1 \ll \varepsilon_{b}^{2} \ll \alpha^{2},
$$

suits to the problem, when $\alpha \gg 1$, and (8) holds.

Now with respect to the wave spectrum. The term $\omega_{d}=\Delta k v_{z}-\Delta \omega$ occuring in (35), describes the group velocity dispersion, and it can be represented in the form

$$
\omega_{d}=\Delta k v_{z} \simeq L^{-1} v_{z}
$$

for $v_{z} / v_{g r} \gg 1$; and,

$$
\omega_{d}=\Delta \omega \simeq T^{-1}
$$

for $v_{z} / v_{g r} \ll 1$.

Note that these representations are tantamount to the SLR and TLR of wave packet, respectively.

We are interested in how relativistic and stochastic effects modify the property of particle acceleration in the case corresponding to particle motion in a SL wave packet.

In the ultra-relativistic limit, both conditions, (37) and (39), are valid, and $v_{z} \simeq 1$, consequently, from (35) the following expression results

$$
\Delta \varepsilon \simeq \varepsilon_{r}^{2}\left(\omega_{B} L\right)^{-1}
$$

in which the relation $\Delta k \simeq 1 / L$ has used.

Resonance wave-particle interaction leads to a widening of the own resonance energy level. Denote by $\delta \varepsilon=\varepsilon-\varepsilon_{r}$ the width of the resonance level.

Integrating equations of motion (30) and (31) with the help of invariant of motion (33), we estimate the level width

$$
\begin{aligned}
& \delta \varepsilon \simeq k \alpha^{1 / 2} \varepsilon_{r}^{-1 / 2}\left(A_{k} / m\right) \Delta t \\
& \Delta t=L / v_{z}, \quad v_{z} \simeq 1
\end{aligned}
$$


Now the criterion defining the allowed domain of random motion in energetic space can be written as $\delta \varepsilon \geq \Delta \varepsilon[10]$, [9] or, in the explicit form,

$$
\varepsilon \leq \varepsilon_{b}=\left(\alpha^{3 / 2}(k L)^{2}\left(A_{k} / m\right) / 2\right)^{2 / 5}
$$

which follows from the two above results, (41) and (42). Formula (43) predicts that the upper value of energetic spectrum depends on the magnitude of wave field as $A^{2 / 5}$.

For describing stochastic behavior of relativistic electron in a TL wave packet, we use the conditions (8), (40) and (35), which correspond to the particular physical situation. Now substituting (40) into (35) we find the interval between the two adjacent resonance states,

$$
\Delta \varepsilon \simeq \varepsilon_{r}^{2}\left(\omega_{B} L\right)^{-1} .
$$

Then with the help of equations of motion (31) and (32) we evaluate the level width

$$
\delta \varepsilon \simeq \omega_{B} T\left(A_{k} / m\right) / 2 \varepsilon_{r} .
$$

Now the condition of resonance overlap, $\delta \varepsilon \geq \Delta \varepsilon$ yields the upper bound of the energetic spectrum,

$$
\varepsilon_{b}=\left(\alpha^{2} \omega^{2} T^{2} A_{k} / m\right)^{1 / 3}
$$

Our analysis infers the possibility of self-generated, chaotic behavior of the system in some region of energetic space. It is easy to see that the structure of resonance energy space mentioned above corresponds to quantized energy space, when each resonance state is assigned the own energy level. The interval between adjacent energy levels caused by the group velocity dispersion depends on the particle energy as $\Delta \varepsilon \propto \varepsilon^{2}$. On the other hand, the width of each energy level is governed by the resonance waveparticle interaction, which becomes weak at very high energies of the particle. Thus the overlap criterion determines the allowed stochastic heating domain. We will carry on our advanced studies of stochastic motion in the next sections.

\section{Relativistic particle dynamics in a coherent wave packet}

We specify first the wave spectrum of a packet. Let us assume that the wave packet is given by (27). Thus, we will consider a relativistic electron motion in the space-like packet (SLP) of the whistler mode waves. In this approach dropping the subscript " 0 " we write down the equations of motion (15) and (16) in the form:

$$
\begin{array}{ll}
\dot{p} & =k A \sqrt{2 m \omega_{B} I} H_{0}^{-1} \sin \psi \sum_{n \in Z} \delta(\zeta-n), \\
\dot{I} & =\sqrt{2 m \omega_{B} I} H_{0}^{-1} A \sin \psi \sum_{n \in Z} \delta(\zeta-n), \\
\dot{z} & =p H_{0}^{-1}, \quad \dot{\theta}=\omega_{B} m H_{0}^{-1} \\
H_{0}(p, I) & =\sqrt{m^{2}+p^{2}+2 m \omega_{B} I} \\
\dot{\psi} & =\omega(p, I)=k p H_{0}^{-1}+\omega_{B} m H_{0}^{-1}-\omega .
\end{array}
$$


Here $A, \omega$ and $k$ are the magnitude, frequency, and wave number of the fundamental (characteristic) mode, $\zeta=(z / L), L$ is the characteristic spacescale, $\delta_{n} \equiv \delta(\zeta-n), \quad \delta(\cdot)$ is the Dirac delta function, and $Z$ denotes the set of all integers.

The evident symmetries of rotation, $\psi \rightarrow \psi \exp \left(i \psi_{0}\right), \psi_{0}$ is an arbitrary constant, and a translation, $\{p, I\}(\zeta) \rightarrow\{p, I\}(\zeta+1)$, allow us to represent dynamics of the system as certain iterative process by identification of the planes $n \zeta$ and $(n+1) \zeta$. The group symmetry is realized first as the invariant of motion,

$$
p-k I=i n v .
$$

By means of (36), we write down expression (52) as

$$
\varepsilon_{z}-\alpha^{-1} \varepsilon_{t}^{2}=0, \quad \alpha=\left(2 \omega_{B} / \omega\right) v_{p h}
$$

where the constant of integration has chosen equals zero. In view of (53) the number of dimensions reduces to two. Let the variables $z$ and $p$ will be the represented pair.

Now the explicit form of iteration system is to be found. Denote by $T M$ the tangent fibering. There exits the map $\bar{v}: M \rightarrow T M$, such that the field of vectors has the form

$$
\bar{v}=k \frac{\sqrt{2 m \omega_{B} I}}{H_{0}} A \sin \psi \sum \delta_{n} \frac{\partial}{\partial p}+p H_{0}^{-1} \frac{\partial}{\partial z} .
$$

Then each element of $\bar{v}$ is assigned the 1-form, so that the field of 1 -forms may be written as

$$
\tilde{v}=-k \frac{\sqrt{2 m \omega_{B} I}}{H_{0}} A \sin \psi \sum \delta_{n} \tilde{d} z+p H_{0}^{-1} \tilde{d} p
$$

The notations $\partial / \partial x^{i}$ and $\tilde{d} x^{i}$ are employed here for the orthornormal coordinate basis of vectors with algebra mentioned above, and for the dual basis of 1-forms, such that

$$
\tilde{d}^{i} \frac{\partial}{\partial x^{i}}=\delta_{i}^{i \prime}=\left\{\begin{array}{ll}
1, & i=i^{\prime} \\
0, & i \neq i^{\prime}
\end{array},\right.
$$

where $\left(x^{i}\right)=(p, z)$.

It is well-known that section of the field of 1 -forms, $\tilde{v}(\bar{v})=0$, on a submanifold in $M$ is equivalent to solution of equations $(48,49)$ ( Schutz, 1982).

Then direct integrating the equation $\left(-k \sqrt{2 m \omega_{B} I} H_{0}^{-1} A \sin \psi \sum \delta_{n} \tilde{d} z+p H_{0}^{-1} \tilde{d} p\right)(\bar{v})=0$ along with (53) yields

$$
\left(p_{n+1} / m\right)^{3 / 2}=\left(p_{n} / m\right)^{3 / 2}+(3 / 2) \alpha^{1 / 2} N(A / m) \sin \psi_{n}, \quad N=[k L],
$$

where $N$ is the characteristic number of modes in the wave packet, [] denotes the integer parts.

Taking into account that the timescale of the problem is $T=L / v_{z}$, from (51) follows the equation $\psi_{n+1}=\psi_{n}+\omega\left(p_{n+1}, I_{n+1}\right) T$, which describes the phase shift acquired by particle. This equation may be written in the explicit form as

$$
\psi_{n+1}=\psi_{n}+N\left(1+\frac{\alpha m}{2 p_{n+1}}\left(1-\frac{\omega}{\omega_{B}} \sqrt{1+\left(\frac{p_{n+1}}{m}\right)^{2}+\alpha \frac{p_{n+1}}{m}}\right)\right)(\bmod 2 \pi)
$$


It is readily seen, if the inequality $\varepsilon^{2} \gg 1$ is valid, the set of equations (56) and (57) can be transformed into the map:

$$
\begin{aligned}
u_{n+1} & =u_{n}+Q \sin \psi_{n}, \\
g^{n}: & \\
\psi_{n+1} & =\psi_{n}+\left(3 \pi^{5 / 3} / 2 Q\right)\left|u_{n+1}\right|^{-2 / 3} \operatorname{sgn} u_{n+1}(\bmod 2 \pi),
\end{aligned}
$$

written in the perception,

$$
u=\pi\left(\frac{\varepsilon}{\varepsilon_{b}}\right)^{3 / 2}, Q=\frac{3 \pi}{4}\left(\frac{\alpha}{\varepsilon_{b}}\right)^{3 / 2} N b, \quad \varepsilon_{b}=\alpha\left(\frac{1}{4} N^{2} b\right)^{2 / 5}
$$

where $u_{n+1}, \psi_{n+1}$ and $u_{n}, \psi_{n}$, are respectively the values of the normed momentum and phase at times $(n+1) \zeta$ and $n \zeta$.

In writing (58) we have used the relationship between fields $A^{w}$ and $B^{w}$,

$$
A / m=\alpha b / 2, \quad b=B^{w} / B,
$$

which follows from equations (3).

The quantity $A / m$ has a clear physical meaning: it is the dimensionless representation of the ratio of the wave field work on one wavelength to the particle rest energy.

Now we describe a relativistic particle motion in a time-like wave packet (TLP).

In this case the Hamiltonian of the problem becomes

$$
H(p, z ; I, \theta ; t)=H_{0}(p, I)+\frac{\sqrt{2 m \omega_{B} I}}{H_{0}} A \cos \psi \sum \delta(\tau-n),
$$

where we used the TLR of wave packet given by (25). Here $\delta(\tau-n)$ is the Dirac function, $\tau=[t / T]$, and $T$ is the characteristic timescale.

The associated with (61) equations of motion, written on framed manifold with the preceeding notation are

$$
\begin{aligned}
& \dot{p}=k \frac{\sqrt{2 m \omega_{B} I}}{H_{0}} A \sin \psi \sum \delta(\tau-n), \quad \dot{I}=\frac{\sqrt{2 m \omega_{B} I}}{H_{0}} A \sin \psi \sum \delta(\tau-n) \\
& \dot{z}=p H_{0}^{-1}, \quad \dot{\theta}=\omega_{B} m H_{0}^{-1} \\
& \dot{\psi}=k \dot{z}+\dot{\theta}-\omega
\end{aligned}
$$

We retain in (63) only the terms of leading order.

These equations can be transformed into a map by identification of the planes $n T$ and $(n+1) T$ as follows.

At first, one must note that the invariant of motion like that (53), lowering the number of degrees of freedom, is retained. Applying the invariant to $(62-64)$ ), we integrated these equations to find the coupled pair of nonlinear difference equations,

$$
\begin{aligned}
& p_{n+1} / m=p_{n} / m+(N \alpha b / 2) \sin \psi_{n}, \quad N=[\omega T], \\
& \psi_{n+1}=\psi_{n}+\frac{1}{2} \sqrt{\alpha} N \sqrt{\frac{m}{\left|p_{n+1}\right|}} \operatorname{sgn} p_{n+1}(\bmod 2 \pi),
\end{aligned}
$$

where the variables $p_{n}, \psi_{n}$ are taken at $t=n T$. 
In the representation

$$
\pi\left(\frac{\varepsilon}{\varepsilon_{b}}\right)^{2}=u, \quad Q=\frac{\pi \alpha^{2} b N}{2 \varepsilon_{b}^{2}}
$$

where

$$
\varepsilon_{b}=\frac{1}{2} \alpha\left(N^{2} b\right)^{1 / 3}
$$

equations (65) go over into a map, $G^{n}$,

$$
\begin{aligned}
& u_{n+1}=u_{n}+Q \sin \psi_{n}, \\
G^{n}: & \\
\psi_{n+1} & =\psi_{n}+2\left(\pi^{3 / 2} / Q\right)\left|u_{n+1}\right|^{-1 / 2} \operatorname{sgn} u_{n+1}(\bmod 2 \pi),
\end{aligned}
$$

that is suitable for sequential analysis.

The closed set of equations (68) is a measure-preserving map expressed in the terms of the canonical pair of variables $\psi, u$. It is obvious, $g^{n}$ as well as $G^{n}$ inherit the canonical structure of the basic equations. Note that the maps $g^{n}, G^{n}$ are formalyzed by the essentially different transformations (59) and (66). In the following we refer to (58) and (68) as corresponding to the SLR case and the TLR case, respectively.

\section{Stochastic attractors of the system}

The maps $g^{n}$ and $G^{n}$, or rather the families of maps depending upon the parameter $Q$, become suitable for sequential analysis. So thereafter we deal with high-frequency heating relativistic particles, expressed in terms of the discrete groups of automorphisms $g^{n}$ and $G^{n}$, that act on smooth manifold. Denote by

$$
J=\frac{\partial\left(u_{n+1}, \psi_{n+1}\right)}{\partial\left(u_{n}, \psi_{n}\right)}
$$

the Jacobi matrix of these maps. It is important to note that the Jacobian of (58) is equal to one, therefore, $g^{n}$ and $G^{n}$ have a structure of the differentiable area-preserving maps. It stands to reason that $u$ and $\psi$ are the canonical pair of variables.

We now study the behavior of this dynamic system by computational analysis. We have numerically integrated equations (58) and (68) for several different values of $Q$ from $10^{-3}$ to 0.1 . Figure 1 shows some our results computed for map (58) after $\left(10^{6}-10^{4}\right)$ iterations for one trajectory in the $(u, \psi)$ phase space. Shown in Figure 2 is the phase space of $G^{n}$. The initial conditions were chosen in a random fashion and corresponded to the region of small values of $(u, \psi)$.

We visual impression of the figures indicates that the phase curves are likely stochastic orbits. So, while smooth curves, piecewise smooth curves, and curves with finite length all have topological dimension one, the phase curves constructed by iteration function systems $g^{n}$ and $G^{n}$ have seemingly dimension close to two. Of course, to clarify this issue, we need to establish that dynamics of the system is realized on stochastic (strange) attractor. Let us consider a pair $\left(M, g^{n}\right)$ and/or a pair $\left(M, G^{n}\right)$, where $M$ be a smooth manifold, and $g^{n}$ and $G^{n}$ are the differentiable area-preserving maps, and 
either of these pairs determine the phase flow on $M$. From (58) and (68) follow that these pairs are invariable under the transformation

$$
\psi \rightarrow-\psi, \quad u \rightarrow-u
$$

and the inversion of a point with respect to a circle $\psi(\bmod 2 \pi) \in S$. On that ground, we define the following equivalence rules:

$$
(-\pi, u) \sim(\pi, u), \quad(\psi,-\pi) \sim(\psi, \pi)
$$

where $\sim$ stands for the equivalence sign.

The identification of these points is indicated by the arrows in Figure 1.

The equivalence rules (71) allow us to represent phase space of the system as the quotient space having the quotient topology of torus, $T^{2}=S \times S, \quad \psi(\bmod 2 \pi) \in S, u \in S[23]$.

Now we discuss the local topology considering a Jacobi matrix given by (69). Denote through $\lambda_{1}$ and $\lambda_{2}$ the eigenvalues of the matrix $J$. Recalling that local structure is determined by the proximate neighbourhoods of fixed points, we have from (69)

$$
\begin{aligned}
& \operatorname{det} J=\lambda_{1} \cdot \lambda_{2}=1, \\
& \operatorname{tr} J=\lambda_{1}+\lambda_{2}=2+\left(\pi^{5} u^{-5}\right)^{1 / 3},
\end{aligned}
$$

for the SLR case; and

$$
\begin{aligned}
& \operatorname{det} J=1, \\
& \operatorname{tr} J=2+\pi^{3 / 2}|u|^{-3 / 2},
\end{aligned}
$$

for the TLR case. Here $\operatorname{det} J$ and $\operatorname{tr} J$ denote the determinant and the trace of this matrix, respectively.

As pointed out in [11] the condition

$$
|\operatorname{tr} J|=3
$$

corresponds to a topological modification of a phase space, and its validity implies that the manifold have topology of a hyperbolic torus. Thereupon from (76) subject to (73), and/or (75) we find

$$
\lambda_{1}=\frac{3+\sqrt{5}}{2}, \quad \lambda_{2}=\frac{3-\sqrt{5}}{2} .
$$

Since $\lambda_{1}$ and $\lambda_{2}$ are the dynamic characteristics such that $\lambda_{2}>0, \lambda_{1}>1$, and the ratio $\lambda_{1} / \lambda_{2}$ is an irrational number, therefore the map $\left(\psi_{n}, u_{n}\right)=g^{n}\left(\psi_{0}, u_{0}\right),\left(\psi_{0}, u_{0}\right)$ is an initial point of phase curve, forms a stochastic phase flow with the mean rate of a loss of infomation, $K$,

$$
K=\ln \lambda_{1}
$$

$K$ is the Kolmogorov entropy.

It is clear, the phase flow $\left(M, G^{n}\right)$ possesses just the same properties.

Present conditions (73), (75) and (76) ensure that the relation

$$
\left|u_{b}\right|=\pi
$$


take place, and it determines the upper bound of $\{u\}$.

Like that the set $\{u, \psi\}$ is a compact, whose structural stability is determined by the fractal dimension, $d_{f}$

$$
d_{f}=1-\ln \lambda_{1} / \ln \lambda_{2}, d_{f}=2 .
$$

We call any compact a probabilistic fractal if its topological dimension is less than $d_{f}$ and $K>0$ [12]. In our case both these conditions are valid.

As seen in Figures 1 and 2 for a given values of $Q$ the stochastic region extends to values of $u$ predicted by above equation (79). We know, that $u$ depends on the parameters of this problem as given by (73) and (75). By that condition (79) determines in itself an equivalence class in the $Q$-parametric space.

Considering $(73,59)$ and $(53)$, from $(79)$ two equations result

$$
\begin{aligned}
\varepsilon_{b} & =\alpha\left(\frac{1}{4} b N^{2}\right)^{2 / 5} \\
\varepsilon_{b} & =\left(\frac{1}{2} \alpha\left(\omega_{B} / \omega\right)^{2} N^{2} b\right)^{1 / 3} \simeq \frac{1}{2} \alpha\left(N^{2} b\right)^{1 / 3},
\end{aligned}
$$

which determine the upper values of the energy spectra for the SLR- and TLR cases, respectively. Note the dependence $\varepsilon_{b}$ on the driving field $b$ is weak enough. This agrees with both the numerical solutions and the results of qualitative analysis.

Finally, it should be noted that the phase flows, $\left(M, g^{n}\right)$ and $\left(M, G^{n}\right)$ are structural stable and typical because $d_{f}$ and $K$ be an invariants, and $\sup \{\varepsilon\}$ smoothly depends on the driving amplitude $b$, which is the controlling parameter of the system. Phase flows with such properties are said to be the stochastic (strange) attractors. Certainly the flows $\left(M, g^{n}\right)$ and $\left(M, G^{n}\right)$ be such attractors as was to be proved.

At last, the achieved fractal measure $d_{f}=2$ infers that points of phase curve evenly fill all obtainable phase space, or in other words, all states of our dynamic system are equivalent. This issue is supported by simulation in Figure 3 on which is represented a joint probability density, $\rho(\psi, u)$. The following algorithm was used for computing $\rho$. We partition all the phase space on the identical cells with the mesh size $\Delta \psi \Delta u=(2 \pi / 40) \times(2 \pi / 30)$. Like that $\rho(\psi, u)$ is proportional to the number of phase points in the element of phase space, $\Delta \psi \Delta u$. Figure 3 reveals that diffusion in the phase angle is very fast. Significant phase angle diffusion occurs on timescales order of tens of $T$, where $T$ is the step of one iteration. On these timescales, the change in $u$ is smaller (the numerical calculations indicate the characteristic time for establishing the uniform distribution in $u$ is propotional to $T / Q^{2}$ at $Q \ll 1$ ), therefore, the variable $u$ is a slow varying coordinate on the strange attractor. Statistical aspect of the problem will be studied in more detail in Sec.6.

\section{Particle stochastic heating}

Particle dynamics in random electromagnetic fields is known to be described by the quasilinear theory (QLT) [25]. The QLT approach, in particular, has been employed to deal with diffusion of electron in the turbulent field of whistler waves packet [22]. 
In coherent electromagnetic fields, on the other hand, the particle dynamics is not described by this theories. The nature of the diffusion in this case is the stochastic dynamics of particles, when the motion along stochastic trajectories gives rise to the so-called deterministic diffusion [1]. General mathematical and physical aspects of this problem have been discussed, for instance, in the reviews [24]; [10].

The purpose of the present paper is to investigate electron dynamics in coherent packet of whistler mode waves. We have employed here the method in which the perturbation of particles orbits are included into a Fokker-Planck-Kolmogorov (FPK) equation [1].

In the present section, we shall exploit the canonical Hamiltonian structure that has been developed above. Thus, the drift kinetic equation follows at once since the canonical structure of equations of motion (58) and (68) has been established. As shown above, the maps $g^{n}$ and $G^{n}$ act on any strange attractor therefore the distribution function (probability density) $f(u ; t)$ on the attractor obeys the Fokker Planck - Kolmogorov (FPK) equation

$$
\frac{\partial f(u ; t)}{\partial t}=\frac{1}{2} \frac{\partial}{\partial u} D \frac{\partial f}{\partial u}
$$

which holds if $Q \ll 1$ (Lichtenberg, 1983).

Here $D$ is the conventional diffusion coefficient in phase space,

$$
D=<\left(u_{n+1}-u_{n}\right)^{2}>T^{-1},
$$

in which $\left(u_{n+1}-u_{n}\right)$ is substituted from (58) or (68), and $<\cdot>$ denotes the phase average, $T$ is the timescale of maps $g^{n}$ or $G^{n}$. The function $f(u, t)$ belongs to the space of all differentiable functions supported in $[-\pi, \pi]$, and the functional

$$
\int_{-\pi}^{\pi} f(u, t) d u=1
$$

is the condition of normalization.

First by means of $g^{n}$ ( or $G^{n}$ ) we calculate by formula (84) the diffusion coefficient

$$
D=Q^{2} / 2 T \text {. }
$$

Then, making use of result (79) proved above, along with (83) and (86), we evaluate the characteristic time for redistribution $u$ over the spectrum

$$
t_{d} \simeq u_{b}^{2} / D=2 T(\pi / Q)^{2} .
$$

A objective of this study is to determine the time-independent distribution function and the rate of heating. This requires a solution of the FPK equation together with a normalization (85). Thus a solution of the FPK equation along with the boundary condition $f(-\pi)=f(\pi)$ in the limiting case $t \geq t_{d}$ may be given in the form of the uniform distribution

$$
f(u)=(2 \pi)^{-1}, \quad u \in l, \quad l=(-\pi, \pi)
$$

for independent random variable $u$ with mean zero. 
Now, it becomes relevant to determine how the system evolves in time at $t \leq t_{d}$. We newly exploit FPK equation with $f(u)$ and its derivative $\partial f / \partial u$ vanishing at the boundary. We introduce the moment $\left\langle u^{2}\right\rangle=\int_{-\pi}^{\pi} d u u^{2} f(u)$, multiply equation (83) by $u^{2}$, and integrate the resulting equation over $u$ to obtain

$$
\frac{d<u^{2}>}{d t}=D
$$

Equations (83-89) describe two physically different situations: an electron motion in the SL wave packet, and, that respectively, in the TL packet.

We know, that the variables $u$ and $\varepsilon$ are related by (59) and (66). This allows us to attach all possible states of $\varepsilon$ a probabilistic measure, namely, the probability density, $f(\varepsilon, t)$, which is associated with $f(u, t)$ via the measure-preserving point transformation

$$
f(\varepsilon, t)=f(u, t)(d u / d(\varepsilon)) .
$$

Representing our results we start with the SLR case. In the case the variables $u$ and $\varepsilon$ are associated by relation (59).

Then from equations (88) and along with the norming

$$
\int_{0}^{\varepsilon_{b}} f(\varepsilon, t) d \varepsilon=1
$$

we derive the steady-state distribution

$$
f(\varepsilon) d \varepsilon=\frac{3}{2} \varepsilon_{b}^{-3 / 2} \varepsilon^{1 / 2} d \varepsilon, \quad\left\{\varepsilon \in R_{+} \mid 0 \leq \varepsilon \leq \varepsilon_{b}\right\},
$$

for the random variable $\varepsilon$ with the mean value $\langle\varepsilon\rangle=0.6 \varepsilon_{b}$, and the relative standard deviation

$$
\frac{\sqrt{\left.<\varepsilon^{2}>-<\varepsilon\right\rangle^{2}}}{\sqrt{<\varepsilon^{2}>}}=0.4
$$

Above equations (91) and (92) describe the state density and level of fluctuations in the energetic spectrum, $\varepsilon_{b}$ is the upper value of this spectrum given by (82).

Then we used relations (59) in equations (89) and (87) to find the rate of diffusion,

$$
\frac{d<\varepsilon^{3}>}{d t}=D, D=\frac{\alpha^{3} N^{2} b^{2}}{16 T}, t<t_{d}
$$

and the characteristic time of diffusion in energy,

$$
t_{d}=16 T\left(N / 64 b^{2}\right)^{2 / 5}
$$

The distribution function $f(u, t)$ in the TLR case obeys also the FPK equation as consequence of the fact that the equations of motion, expressed in coordinates $(u, \psi)$ reflect the underlying canonical Hamiltonian structure of equations (68).

Now we use the dynamical equations (68), written in representation (66) and the local transformation (90), to derive equations, governing diffusion in energetic space. Thus the energy spectrum of particle follows from formula (88)

$$
f(\varepsilon) d \varepsilon=\frac{2}{\varepsilon_{b}^{2}} \varepsilon d \varepsilon
$$


where $\varepsilon_{b}$ is given by $(82)$. We have respect to equations $(83,84)$ and $(87)$ to find the equations, describing evolution of the energy spectrum

$$
\begin{aligned}
& \frac{\partial}{\partial t} f(\varepsilon, t)=\frac{1}{2} \frac{\partial}{\partial \varepsilon} D(\varepsilon) \frac{\partial}{\partial \varepsilon} f(\varepsilon, t) \\
& D(\varepsilon) \quad=\frac{\alpha^{4} N^{2} b^{2}}{32 T \varepsilon^{2}} \\
& \frac{d<\varepsilon^{4}>}{d t}=\frac{\alpha^{4} N^{2} b^{2}}{16 T}, t<t_{d} \\
& t_{d}=\frac{1}{2} T(N / b)^{2 / 3}
\end{aligned}
$$

Notice expressions (93) and (98) describe a stochastic process differs essentially from the Brownian-like diffusion. Furthermore solutions of equations (93) and (98) predict qualitatively different behavior of the system. Thus in the SLR case the particle energy grows with time as $t^{1 / 3}$; in another case the energy growth is proportional to $t^{1 / 4}$.

Now we describe the effects associated with the stochastic heating of particle. First we discuss the pitch angle distribution over particle energies. Denote by $\chi_{p}$ the pitch angle and let $\chi$ is its complementary angle. Then on account of $A^{w} / m \ll 1$ we have

$$
\tan \chi=\frac{v_{z}}{v_{t}} \simeq \frac{\varepsilon_{z}}{\varepsilon_{t}}
$$

Taking account of invariant of motion (53) we derive the following dependence

$$
\tan \chi=\left(\varepsilon_{z} / \alpha\right)^{1 / 2}
$$

Thereupon correspondence (91) along with the measure-preserving transformation $f(\chi) d \chi=f(\varepsilon) d \varepsilon$ yield the time-independent pitch angle distribution $f(\chi)$, namely,

$$
\begin{aligned}
& f(\chi) d \chi=3\left(\alpha / \varepsilon_{b}\right)^{3 / 2}\left(1+\tan ^{2} \chi\right) \tan ^{2} \chi d \chi, \quad \chi \in\left(-\chi_{b}, \chi_{b}\right), \\
& \tan \chi_{b}=\left(\varepsilon_{b} / \alpha\right)^{1 / 2},
\end{aligned}
$$

and its dependence on the particle energy

$$
f(\chi(\varepsilon))=3 \frac{\varepsilon^{2}}{\varepsilon_{b}^{3 / 2} \alpha^{1 / 2}} .
$$

Here $\varepsilon_{b}$ is given by (81). A function given by (102) is the concave symmetric function of $\chi$, having the maximum at $\chi=\pi / 2\left(\chi_{p}=0\right)$, its derivatives tend to infinity as $\chi \rightarrow \pm \pi / 2$. This function describes the so-called U-like distribution.

In another case under condition (38) we write down expression (100) simply as

$$
\begin{aligned}
& \chi \simeq \varepsilon / \alpha, \\
& \chi \in\left(-\chi_{b}, \chi_{b}\right), \quad \chi_{b}=\varepsilon_{b} / \alpha,
\end{aligned}
$$


where $\varepsilon_{b}$ is given by (82). Then we find the pitch angle distribution function,

$$
f(\chi)=\begin{array}{r}
|\chi| / \chi_{b}^{2}, \quad|\chi| \leq \chi_{b} \\
0, \text { elsewhere }
\end{array}
$$

for the random variable $\chi$ with mean zero, and its dependence on the particle energy

$$
f(\chi(\varepsilon))=\alpha \frac{\varepsilon}{\varepsilon_{b}^{2}}
$$

Now the equation governing diffusion in pitch angle may be derived via the measurepreserving transformation $f(\chi, t)=f(\varepsilon, t) d \varepsilon / d \chi$ and the relation $\chi=\varepsilon / \alpha$. For $f(\chi, t)$ satisfying these conditions on $\left[-\chi_{b}, \chi_{b}\right]$, we write down the following FPK equation

$$
\begin{aligned}
& \frac{\partial}{\partial t} f(\chi, t)=\frac{\partial}{\partial \chi} D(\chi) \frac{\partial}{\partial \chi} f(\chi, t) \\
& D(\chi)=\frac{N^{2} b^{2}}{32 T \chi^{2}}
\end{aligned}
$$

From these equations we find the timescale for scattering,

$$
t_{s}=32 T \frac{\chi_{b}^{4}}{N^{2} b^{2}}=T(N / b)^{2 / 3}
$$

and the scattering rate,

$$
\frac{d}{d t}<\chi^{4}>=\frac{N^{2} b^{2}}{16 T}, \quad t<t_{s}
$$

According as (99) and (110), we note the timescales for heating and scattering are comparable.

Next we show that the stochastic heating is accompanied by a spatial diffusion of electrons across a homogeneous magnetic field. Indeed, because of the one-one correspondence $r=\varepsilon_{t} / \omega_{B}$ and the invariant of motion (53), the distributions of electrons over $r$ and $\varepsilon$ are related uniquely by the relation $f(r, t)=f(\varepsilon, t) d \varepsilon / d r$. It means that $f(r, t)$ obeys the FPK equation

$$
\begin{aligned}
\frac{\partial}{\partial t} f(r, t) & =\frac{1}{2} \frac{\partial}{\partial r} D_{t}(r) \frac{\partial}{\partial r} f(r, t), \\
D_{t}(r) & =\frac{\alpha^{4} N^{2} b^{2}}{32 T \varepsilon^{2} \omega_{B}^{2}},
\end{aligned}
$$

which follows from equation (96) at once.

The function $f(r, t)$ is supported on $\left[r_{0}, r_{b}\right]$, where $r_{0}=1 / \omega_{B}$ and $r_{b}=\varepsilon_{b} / \omega_{B}$, and $D_{t}$ is the coefficient of diffusion of electrons across a magnetic field.

One should be noted, that a classical Brownian-like diffusion process predicts that the mean square displacement grows linearly with time, and its rate is given by $D_{t}^{B} \simeq \nu_{e i}<r^{2}>$ representing the Bohm-like diffusion coefficient $\left(\nu_{e i}\right.$ is the frequency of collisions). 
Note that $D_{t}^{B}$ has the strong dependence on $B$ (as $B^{-2}$ ), while the coefficient of diffusion given by (113) is non-sensitive to change in $B$, and it hinges on $b$ and $\varepsilon$ as $b^{2}, \varepsilon^{-2}$. Equations (112 and (113) are correct, if the inequality $r_{b} / L \ll 1$ holds. In view of equation (82), this requiring can be written in the form $r_{b} / L \simeq\left(\omega / \omega_{B}\right)\left(\varepsilon_{b} / N\right),(\ll 1)$. For typical parameters this condition is satisfied trivially.

We have done the solution of this problem. By way of illustration, we apply the results of our inverstigation to the Jupiter magnetosphere.

\section{Application}

It is known that the Jovian magnetosphere contains high-energy electrons spiralling in strong Jupiter's magnetic field. Thus the existence of Jupiter's radiation belts were confirmed via synchrotron emission measurements by Allen et al [13] and Fischer et al [14]. Of late Bolton et al [15] have been reported about the presence of electrons at energies up to $50 \mathrm{MeV}$ in Jupiter's inner radiation belts. They have been suggested that high energy electrons require substantial acceleration by processes other than adiabatic radial diffusion. The mechanism may be local acceleration of electrons by whistler mode waves, which observed in the Jovian magnetosphere [16].

To check the performance of the method, at first the parameters of the problem are chosen to reflect a typical whistler wave propagating along the $L_{p}=6$ shell, where the electron cyclotron frequency $\omega_{B}=3.3 \cdot 10^{5} \mathrm{~s}^{-1}$, the electron plasma frequency $\omega_{p}=2.2 \cdot 10^{6} \mathrm{~s}^{-1}$, and the wave frequency $\omega=1.5 \cdot 10^{5} \mathrm{~s}^{-1}$. Thus the dispersion relation (8) corresponds, undoubtedly to the problem, and by (8) we find the phase velocity, $v_{p h} \simeq 0.08$ and the wavenumber $k=6 \cdot 10^{-5} \mathrm{~cm}^{-1}$ The theory is based on three parameters, namely, $\alpha, N, b$. First we evaluate $\alpha=2 v_{p h} \omega_{B} / \omega, \alpha=0.32$. Typical magnitudes of whistler mode waves are in range of $10^{-7} \mathrm{G}$, consequently the parameter $b$ is of order $10^{-5}$ [17]. To evaluate the parameter $N=k L$ for the space-like wave packet, we need to estimate the characteristic size of the domain of resonant interaction (the zone of intense wave activity). Thus the characteristic size of the region is of the same order of magnitude as the spacescale of inhomogeneity of a background magnetic field near the Io torus, we have accepted $L=5 \cdot 10^{8} \mathrm{~cm}$. By that the value of $N$ is about $N=3 \cdot 10^{4}$. In the following we will use these values to obtain an estimate of some quantities. First by means of (82) we calculate the upper value of energetic spectrum, $E_{b} \simeq 3.5 \mathrm{MeV}$. Considering that the transit time of the particle through the intense wave activity domain is $T=L / v_{z}$, i.e. $T \simeq 1.67 \cdot 10^{-2} \mathrm{~s}$, we evaluate by (94) the timescale of stochastic diffusion over the energetic spectrum, $t_{d} \simeq 8-9$ hours. Now the effect of pitch angle scattering should be evaluated. From (102) at $\varepsilon_{b}=7$ and $\alpha=0.32$ follows that in this case whistler waves can diffuse electrons in a cone with the vertex angle, $\chi_{v}=2 \chi_{b}$, which is about $156^{0}$. The equation (103) indicates a degree of scattering anisotropy, $\operatorname{deg} A=f(\chi)\left(\varepsilon=\varepsilon_{b}\right) / f(\chi)(\varepsilon=1)$, increases with $\varepsilon$ up to $\operatorname{deg} A \simeq 50$.

Now we apply our results to Jupiter's inner radiation belts. Here we use the following values: $\omega_{B}=2 \cdot 10^{7} \mathrm{~s}^{-1}, \omega_{p}=5.65 \cdot 10^{5} \mathrm{~s}^{-1}, \omega=1.5 \cdot 10^{5} \mathrm{~s}^{-1}$. Thereafter we 
obtain $v_{p h} \simeq 0.95$, and $\alpha \simeq 270$. Now the transit time is $L / v_{g r}$, where $L$ is the lengthscale of inhomogeneity at these locations, $L \simeq 10^{9} \mathrm{~cm}$, therefore $T$ is about $3 \cdot 10^{-2} \mathrm{~s}$. In view of $(3), B^{w}=E^{w} / v_{p h}$, and using a spectral density of $(1-3) \cdot 10^{-11} \mathrm{~V}^{2} \mathrm{~m}^{-2} / \mathrm{Hz}$ obtained by Kurth et al [16] and Hobara et al [18] we find $B^{w} \simeq 1.6 \cdot 10^{-8} \mathrm{G}$. Like that the reference parameters are: $b=1.6 \cdot 10^{-8}$ and $N=[\omega T]=5 \cdot 10^{3}$. Then we calculate by (98) and by (99) the upper value of the particle energy, $E_{b} \simeq 50 \mathrm{MeV}$, and respectively the timescale of establishing the energetic spectrum, $t_{d} \simeq 7-8$ days. These expressions yield the mean rate of heating, namely, $\varepsilon_{b} / 2 t_{d}=\alpha b / T, \varepsilon_{b} / 2 t_{d} \simeq 100 \mathrm{eVs}^{-1}$. It is clear the mean heating rate depends linearly on the wave field magnitude, moreover the mean energy acquired by a particle equals to the wave field work on one wavelength multiplied by the ratio of the diffusion time to the characteristic particle transit time through the wave packet. We find that the phase space diffusion leads to a diffusion of electrons across an external magnetic field. Then (113) we calculate the rate the diffusion caused by chaotic motion, $D_{t} \simeq 2 \cdot 10^{7} \varepsilon^{-2} \mathrm{~cm}^{2} / \mathrm{s}$. This process is more effective than a classical Bohm-like diffusion. To show this we use the following simple estimate. Taking plasma density of $10^{2} \mathrm{~cm}^{-3}$ and assuming $v \simeq c$, we obtain from $D_{t}^{B} \simeq \nu_{e i}\left\langle r^{2}\right\rangle$ that $D_{t}^{B} \simeq 5 \cdot 10^{-6} \mathrm{~cm}^{2} / \mathrm{s}$. Thus the diffusion in phase space results in an enchancement of the absolute radial diffusion. Now we respect to the pitch angle scattering. The results (105) and (107) show that the wave packet to effectively scatter electrons in pitch angle leading to establishing the distribution peaked at $\chi_{p} \simeq 74^{0}$ with the degree of anisotropy $\operatorname{deg} A=100$. Of particular importance is that in this energy range, the characteristic scattering time is comparable to the time of diffusion in energy.

Relativistic charged particles lose their energy through the emission of electromagnetic waves. When the dominant loss channels are the synchrotron radiation and the inverse Compton effect, the rate with which a relativistic particle loses its energy is

$$
\dot{\varepsilon}=-\beta \varepsilon^{2}
$$

where $\beta$ is the radiation coefficient.

As a result, we arrive from (114) at the formula

$$
t_{r}=(\beta \varepsilon)^{-1}=5.1 \cdot 10^{8}\left(B_{0} / B\right)^{2} \varepsilon^{-1}, \quad B_{0}=1.0 \mathrm{G},
$$

for the characteristic lifetime of the particle [19].

Comparing $t_{r}$ and the timescale of diffusion over spectrum, $t_{d}$, shows that typically $t_{r} \gg t_{d}$, therefore the effect of emission on stochastic heating is negligibly small. Thus expressions (81) and (82) for upper values of particle energy spectra remain, and changes in the shape of spectra are very little. It is known [19] that wave spectrum of synchrotron emission is characterized by a well defined frequency,

$$
\omega_{r}=\omega_{B} \varepsilon^{2}
$$

and it can be represented with the help of the relation (95) in the form of the uniform distribution

$$
f\left(\omega_{r}\right) d \omega_{r}=\omega_{b}^{-1} d \omega_{r}
$$


where $\omega_{b}=\omega_{B} \varepsilon_{b}^{2}$ is the limiting frequency in the spectrum.

We observe from (116) that the frequencies at which an electron emits the synchrotron radiation is considerable higher than the plasma frequency $\omega_{p}$. It is interesting in that this radiation, providing an information about the energy spectra, can leave the local plasma, and then be detected by an outside observer.

Now to see that our approach correctly interprets the facts, it is necessary to find the range of validity of our results. The equations of motion (56) and (57) in the region of relativistic energies are obtained in the faithful representation, and the ultra-relativistic approximation, $\varepsilon^{2} \gg \max \left\{\alpha^{2}, 1\right\}$, has been employed in deriving (58). According to (81), this inequality may be written in the form

$$
2\left(\omega_{B} / \omega\right) v_{p h} \varepsilon^{-1}<1
$$

Owing to (82) the latter can be transformed into the requiring

$$
b=B^{w} / B>4 N^{-2} \alpha^{-5 / 2}\left(\simeq 8 \cdot 10^{-8}\right) .
$$

In another case electron motion obeys equations (68), obtained in the approximation, $1 \ll \varepsilon^{2} \ll \alpha^{2}$. Taking into account expression (82), we write down this condition as

$$
8 N^{-2} \alpha^{-3}<b<8 N^{-2} \text {. }
$$

By typical condition for Jupiter's inner magnetosphere the above expression gives $10^{-14}<b<4 \cdot 10^{-7}$. These conditions are fairly easy to check. Recalling a spectral density measurements [16] and [18] yield $B^{w} \simeq 10^{-7} \mathrm{G}$, we conclude that (119) and (120) trivially satisfied, therefore, the upper limits for the energetic spectra given by (81) and (82) are correct.

At last, the basic equations are applicable provided $k L \gg 1$, and $\omega_{B} T \gg 1$, and $A^{w} / m \ll 1$, which are typically fulfiled.

It should be noted that the geometry of an ambient magnetic field does not play a role in the given problem, thus the lengthscale of an interaction region is typically smaller than the travelling path, i.e. an ambient magnetic field is always locally an uniform field. To this may be added the analytic model describing the motion of a particle out of the interaction region must include the radial diffusion of electrons conserving the first and second adiabatic invariants.

We conclude that for typical values of the wave field in the Io torus, significant diffusion occurs on timescales of the order of a few hours for electrons with energies up to $3.5 \mathrm{MeV}$. In the inner radiation belts stochastic acceleration of electrons by whistler waves could provide a viable mechanism for energezation of electrons from energies near $1 \mathrm{MeV}$ to $50 \mathrm{MeV}$ over a period of a few days. The results obtained indicated the dependence of maximal electron energy $\varepsilon_{b}$ on the magnitude of wave field $b$ is rather weak, although $\varepsilon_{b}$ increases with increasing $b$.

The obtained energetic spectra have used for evaluating pitch angle distribution functions over different energies. Stochasticity in the pitch angle distribution of relativistic electrons spiralling in a strong magnetic field near Jupiter is appeared as sharp peak near $90^{\circ}$; in relatively weak magnetic field at $L_{p}=6$ electrons are mainly 
scattered by whistler waves almost along the direction of an external magnetic field. The time to scatter a particle into the scattering cone is comparable to the time of diffusion energy.

The electrons involved into a process of stochastic acceleration produce radiation via the synchrotron mechanism. Thus energetic electrons emitting observed hectometric [20] and centimetric [15] synchrotron radiation respectively in Jupiter's outer- and inner radiation belts according to (116) and (117) must have energy comparable to $3.5 \mathrm{MeV}$ and $50 \mathrm{MeV}$. Thereto the observation of synchrotron emissions is obviously evidence for in situ particle acceleration. This is a further confirmation that the model is correctly predicting the main features of the observations.

Chaotic motion driven by wave packet is also responsible for enchanced particle diffusion across an external magnetic field. This result in reasonable agreement with the experimental data.

\section{Summary}

A canonical Hamiltonian approach has been employed to deal with the whistler waveelectron interaction and the stochastic heating of high-energy electrons in magnetized plasmas. The time-like and space-like wave packet representations have used in deriving the equations of motion for relativistic electrons.

The irreversible dynamics on a strange attractor was studied via the FPK equations with a coefficient of diffusion calculated from the equations of motion. The FPK equations describe such macroscopic effects as the stochastic heating, pitch angle scattering and radial drift of plasma particles. The effect of stochasticity on spectrum of synchrotron emission was also evaluated.

The mathamatical structure is realized in the Jovian radiation belt events as follows.

The main feature of Jupiter's radiation belts is its striking stability in a dynamic state far from equilibrium.

On the other hand the results indicate phase flow of our dynamical system is structural stable strange attractor. Chaotic motion on the attractor gives rise to an irreversible process (the so-called deterministic diffusion), which leads actually to establishing an steady-state energy spectra and results in such important and easily observable effects as the stochastic heating and pitch angle scattering of plasma particles. Under conditions typical of this mechanism, the heating region is determined by the boundaries of the attractor and the heating rate is governed by the nature of the kinetics, which in turn depends on the canonical variables on the attractor. The same conditions impose limitations on the timescales of macroscopic effects and feasible extent of heating, so that understanding of this conditions is of great practical interest.

\section{References}

[1] Lichtenberg A and Lieberman M 1983 Regular and Stochastic Motion (New York: Springer) 
[2] Chernikov A A, Tel T, Vattay G and Zaslavsky G M 1989 Phys. Rev. A40 4072-4076

[3] Klimov O V and Tel'nikhin A A 1995 Plasma Phys. Rep. 21 335-338

$\because$

[4] Krotov A S and Tel'nikhin A A 1998 Plasma Phys. Rep. 24 767-771

[5] Nagornykh E and Tel'nikhin A 2002 Proc. 2 th Int. Congress on Plasma Phys. ( Sydney, Australia, ed. by J.S. Falkoner, R.L. Dewar, and J.Khachan Amer. Inst. of Phys. New York) 824-827

[6] Zaslavsky G, Sagdeev R, Usikov D and Chernikov A 1991 Weak Chaos and Quasi-Regular Patterns (Cambridge: Cambr. University Press)

[7] Walker A D 1993 Plasma Waves in the Magnetosphere ( New York: Springer)

[8] Summers D, Thorne R M and Xiao F 1998 J. Geophys. Res. 103 20487-20500

[9] Zaslavsky G 1998 Physics of Chaos in Hamiltonian systems London: ICP)

[10] Chirikov B V 1979 A Phys.Rep. 52 463-487

[11] Arnold V and A Avez 1968 Ergodic Problems of Classical Mechanics (New York: Benjamin)

[12] Mandelbrot B 1982 The Fractal Geometry of Nature (San Francisko: Freeman)

[13] Van Allen J A, Baker D N, Randall B A and Sentman D D 1975 Science 188 459-462

[14] Fischer H M, Pehlke E, Wibberenz G et al. 1996 Sci. 272 856-858

[15] Bolton S J et al. 2002 Nature 415 987-991

[16] Kurth W S, Strayer B D, Gurnett D A and Scarf F L 1985 Icarus 61 497-508

[17] Wykes W J, Chapman S C and Rowlands G 2001 Planet. Space Sci. 49 395-404

[18] Hobara Y, S Kanemaru, and M Hayakawa 1997 J. Geophys. Res. 102 7115-7122

[19] Ginzburg V L 1987 Theoretical Physics and Astrophysics (Moscow: Science)

[20] Ladreiter H P, Zarka P and Lecacheux A 1994 Planet. Space Sci. 42919931

[21] Horne R B and R M Thorne 1998 Geophys. Res. Lett. 25 3011-3013

[22] Kennel C F and Petchek H E 1966 Geophys. Res, 71 1-28

[23] Kosniowski C 1980 First Course in Algebraic Topology (London: Cambr. Univ. Press)

[24] Prigogine I 1980 From Being to Becoming: time and complexity in the physical sciences (San Francisko: Freeman)

[25] Sagdeev R Z and Galeev A A 1969 Nonlinear plasma theory (New York: Benjamin) 
[26] Schuster H 1984 Deterministic Chaos (Weinheim: Physik Verlag)

[27] Schutz 1982 Geometrical methods of Mathematical Physics (Cambridge, London: Cambr. Univ. Press) 

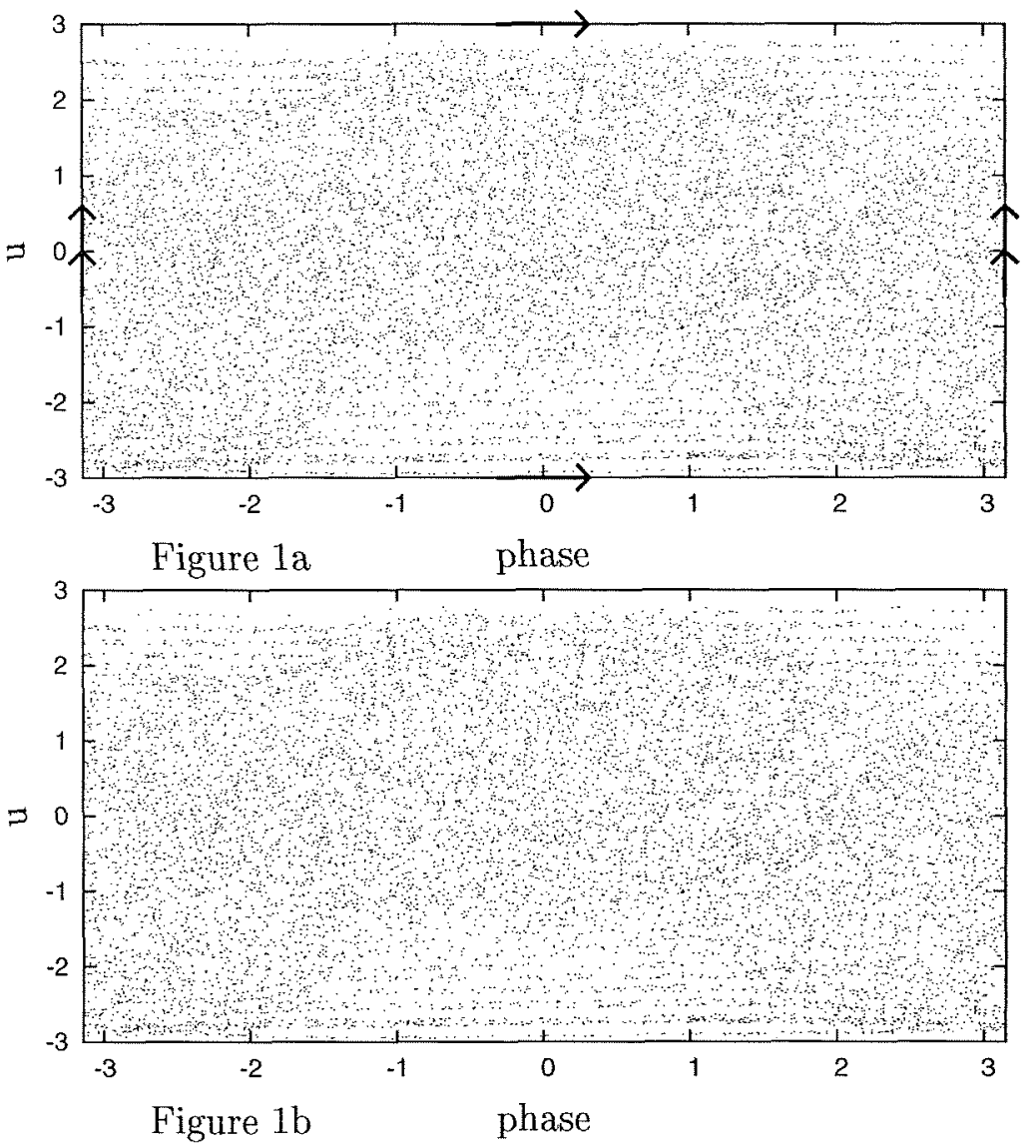

Figure 1. The phase space of the map $g^{n}$. (a) One single trajectory of length $10^{6}$ for $Q=0.01 \pi$. The trajectory was started from the point $u_{0}=10^{-3}, \psi_{0}=10^{-4}$. The identification of points is indicated by the arrows. (b) Close to zero, approximation $g^{n}$ loses its validity. Therefore in the vicinity of $u=0$ we must use the solution of the original equations (56) and (57) expressed in terms of the variables of $u, \psi$ given by (59). The figure shows the boundary of the chaotic region is well approximated by the condition (79). The parameters are the the same as in Figure 1a. 


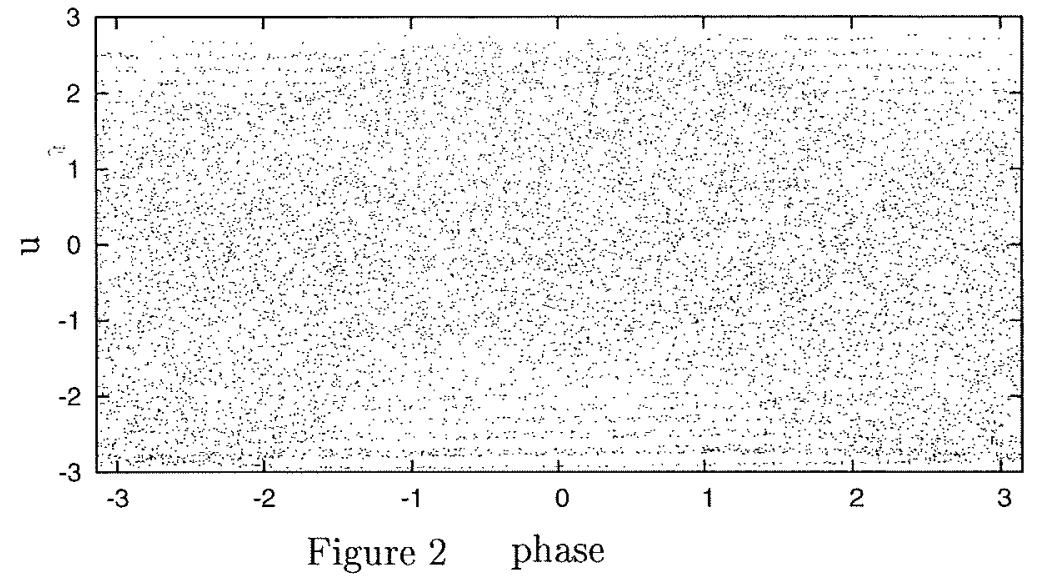

Figure 2. The phase space of the map $G^{n}$. A single trajectory of length $10^{6}$ for $Q=10^{-3} \pi$. The trajectory was started from the point $\left(10^{-4}, 10^{-3}\right)$. 


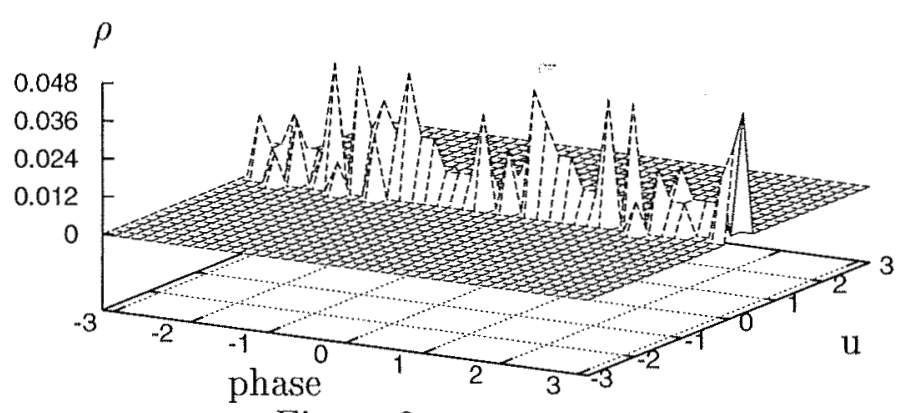

Figure $3 \mathrm{a}$

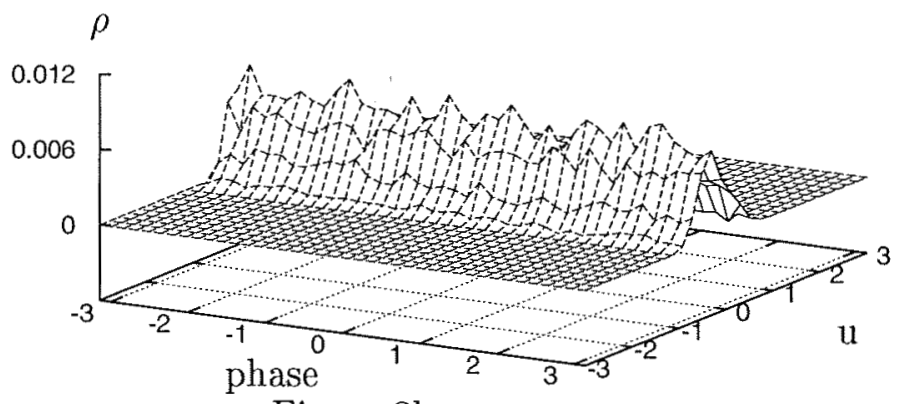

Figure $3 \mathrm{~b}$

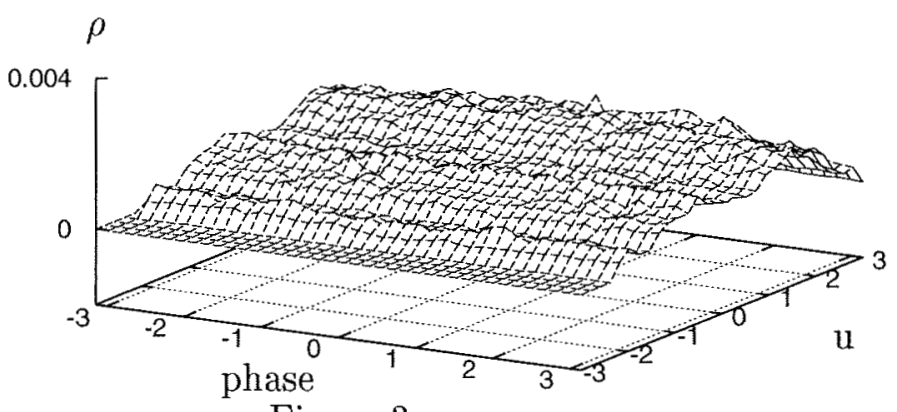

Figure 3c

Figure 3. Joint distribution $\rho(\psi, u)$ computed numerically via $G^{n}$ at several different number of steps. The parameters are the same as in Figure 2. The Figure shows $\rho(\psi, u)$ at times (a) $t=10^{2}$; (b) $10^{4}$; (c) $10^{5}$, respectively. The diagram at $t=10^{2}$ shows uniform distribution over $\psi$ is already established. Figure $3 c$ indicates uniform steady-state distribution, implying that the dimension of the attractor is 2 . 